\title{
Making Graphene Luminescent by Oxygen Plasma Treatment
}

\author{
T. Gokus, ${ }^{\dagger}$ R. R. Nair, ${ }^{\ddagger \star}$ A. Bonetti, $^{\S}$ M. Böhmler, ${ }^{\dagger}$ A. Lombardo, ${ }^{\S}$ K. S. Novoselov, ${ }^{\ddagger}$ A. K. Geim, ${ }^{\ddagger}$ \\ A. C. Ferrari, ${ }^{\S, *}$ and A. Hartschuh ${ }^{\dagger, *}$
}

${ }^{\dagger}$ Chemistry and Biochemistry Department and CeNS, Ludwig-Maximilians-University of Munich, 80539 Munich, Germany, ${ }^{\ddagger}$ Department of Physics and Astronomy, Manchester University, Manchester M13 9PL, U.K., and §Department of Engineering, University of Cambridge, Cambridge CB3 OFA, U.K.

G raphene is at the center of a significant research effort. ${ }^{1}$ Near-ballistic transport at room temperature and high mobility ${ }^{2-4}$ make it a potential material for nanoelectronics, ${ }^{5,6}$ especially for high-frequency applications. ${ }^{7}$ Furthermore, its optical and mechanical properties are ideal for micro- and nanomechanical systems, thin-film transistors, transparent and conductive composites and electrodes, ${ }^{8-10}$ and photonics. ${ }^{11}$ There are two main avenues to modify the electronic structure of graphene. One is by cutting it into ribbons and quantum dots, $, 5,6-12$ and the other is by means of chemical or physical treatments with different gases to reduce the connectivity of the $\pi$ electron network. ${ }^{15,16}$ One of the most popular insulating chemical derivatives is graphene oxide (GO). ${ }^{16}$ Bulk GO solutions and solids do also show a broad luminescence background. ${ }^{17,18}$ Hydrogen plasma was used to controllably and reversibly modulate the electronic properties of individual graphene flakes, turning them into insulators. ${ }^{15}$ Aggressive oxygen treatment was applied to create graphene islands. ${ }^{19}$ However, thus far, no photoluminescence $(\mathrm{PL})$ was seen from individual graphene layers, either cut into ribbons or dots or chemically treated, making graphene integration into optoelectronics still elusive.

Here, we show that PL can be induced in single-layer graphene using an oxygen plasma treatment. PL characteristics are spatially uniform across the flakes and connected to elastic scattering spectra distinctly different from those of gapless pristine graphene. Oxygen plasma can be used to selectively convert the topmost layer when multilayer samples are treated.
ABSTRACT We show that strong photoluminescence $(\mathrm{PL})$ can be induced in single-layer graphene using an oxygen plasma treatment. The PL is spatially uniform across the flakes and connected to elastic scattering spectra distinctly different from those of gapless pristine graphene. Oxygen plasma can be used to selectively convert the topmost layer when multilayer samples are treated.

KEYWORDS: graphene $\cdot$ photoluminescence $\cdot$ optical properties

\section{RESULTS AND DISCUSSION}

Graphene samples are produced by microcleavage of graphite on a silicon substrate covered with $100 \mathrm{~nm} \mathrm{\textrm {SiO } _ { 2 }}{ }^{1}{ }^{1}$ The number of layers is determined by a combination of optical microscopy and Raman spectroscopy. ${ }^{20,21}$ Optical imaging at 473 and $514 \mathrm{~nm}$ is done in an inverted confocal microscope. The beam is reflected by a splitter and focused with a high numerical aperture objective (NA = 0.95). Raman spectra are measured at $514 \mathrm{~nm}$ with a Renishaw micro-Raman spectrometer. The samples are then exposed to oxygen/ argon (1:2) RF plasma (0.04 mbar, $10 \mathrm{~W}$ ) for increasing time ( $1-6 \mathrm{~s})$. The structural and optical changes are monitored by Raman spectroscopy and elastic light scattering. The PL decay dynamics is recorded by timecorrelated single photon counting (TCSPC) upon pulsed excitation at $530 \mathrm{~nm}(2.34 \mathrm{eV})$, with a time resolution of $\pm 3 \mathrm{ps}$. The acquisition time per pixel is of the order of a few tens of milliseconds. The spatial resolution is $\sim 800 \mathrm{~nm}$.

Spatially resolved PL shows bright and localized emission for short treatment times (Figure 1a). For longer times, the $\mathrm{PL}$ is strong and spatially homogeneous (Figure $1 \mathrm{~b}$ ), with a single broad band centered at $\sim 700 \mathrm{~nm}(1.77 \mathrm{eV})$ (Figure 1c). Intense laser excitation with power exceeding $1 \mathrm{~mW}$ leads to photobleaching and a PL maximum blue

\author{
*Address correspondence to \\ acf26@eng.cam.ac.uk, \\ aprpc@cup.uni-muenchen.de, \\ rahul.raveendran-nair@ \\ postgrad.manchester.ac.uk. \\ Received for review September 22, \\ 2009 and accepted November 13, 2009. \\ Published online November 19, 2009. \\ $10.1021 / \mathrm{nn} 9012753$ \\ (c) 2009 American Chemical Society
}




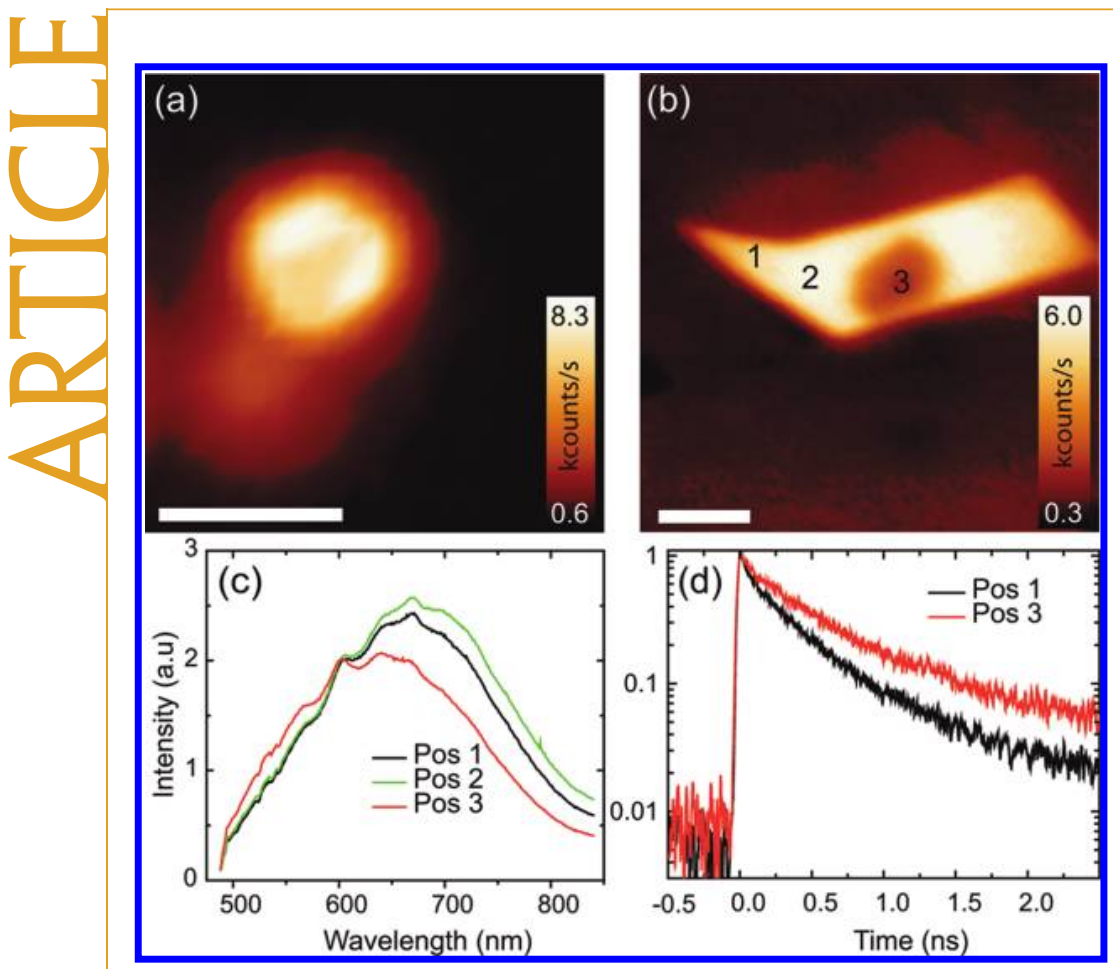

Figure 1. (a) Confocal PL image excited at $473 \mathrm{~nm}(2.62 \mathrm{eV})$ for a graphene sample oxidized for $3 \mathrm{~s}$. Scale bar $=5 \mu \mathrm{m}$. The bright PL spots are spatially localized. (b) Uniform emission after $5 \mathrm{~s}$. Scale bar $=10 \mu \mathrm{m}$. For position 3 in (b), PL is bleached intentionally by intense laser irradiation. (c) Spectra detected at the positions marked in (b) corrected for detector response. These have broad PL centered $\sim 700 \mathrm{~nm}(1.77 \mathrm{eV})$. (d) PL transients detected at the positions indicated in (b). The dynamics can be described by a triple-exponential with decay times 40,200 , and 1000 ps.
Methods). A broad PL background is evident in Figure $2 \mathrm{a}$ for treatment times above $2 \mathrm{~s}$. This is different from the case of the hydrogen plasma treated samples of ref 15 , where no luminescence was observed for the same excitation and detection energy range. Figure $2 \mathrm{a}$ also shows a significant increase of the $D$ and $D^{\prime}$ intensities, and the $D+D^{\prime}$ combination mode at $\sim 2950 \mathrm{~cm}^{-1}$, which requires a defect for its activation. Note that in defected graphene the relaxation of the backscattering condition results in significant broadening of the second-order modes. Defect scattering also broadens the first-order peaks, eventually merging $G$ and $D^{\prime}$ in a single wide $G$ band for treatment times above $1 \mathrm{~s}$.

Figure $2 b$ plots the evolution of the $D$ to $G$ peak intensity ratio, $/(\mathrm{D}) / /(\mathrm{G})$. This first raises and then decreases for increasing time. The $D$ peak intensity is a measure of the number of defects ${ }^{24,25}$ (see Methods). Reference 25 noted that $I(D) / I(G)$ varies inversely with the cluster size $L_{a}$ in poly- and nanocrystalline graphites: $I(D) / I(G)=C(\lambda) / L_{a}$, where $C(514.5 \mathrm{~nm}) \sim 4.4 \mathrm{~nm}^{25,26}$ This is known as the Tuinstra and Koenig relation (TK). TK holds until a critical defect density. ${ }^{24}$ Indeed, ref. 24 noted that, since the $D$ peak requires the presence of six-fold rings, when the network starts losing them, I(D) decreases with decreasing $L_{\mathrm{a}}{ }^{24}$ In this case, $I(\mathrm{D}) / /(\mathrm{G})=C^{\prime}(\lambda) L_{\mathrm{a}}{ }^{2}$ with $C^{\prime}(514.5 \mathrm{~nm}) \sim 0.55 \mathrm{~nm}^{-2} .{ }^{24}$ Combining the latter with $\mathrm{TK}$, we deduce that Figure $2 \mathrm{~b}$ shows a con-

toluminecent graphene (PLG) is complex. The PL transients of Figure $1 \mathrm{~d}$ can be described by a three-exponential decay with lifetimes $\sim 40,200$, and 1000 ps, much longer than those observed in semiconducting nanotubes and amorphous carbon. ${ }^{22,23} \mathrm{Re}$ markably, the PL transients are nearly uniform across the complete spectrum. This implies that spectral diffusion due to energy migration, typical for heterogeneously broadened systems, is absent (see Figure 6 in Methods). We note that PL spectra for pulsed and CW excitation obtained for the same average power are almost identical. This excludes blackbody radiation due to laser heating as the source of the observed emission.

A Raman investigation gives further insights into the evolution from pristine graphene to PLG. Figure 2 plots the Raman spectra and the main fitting parameters (see

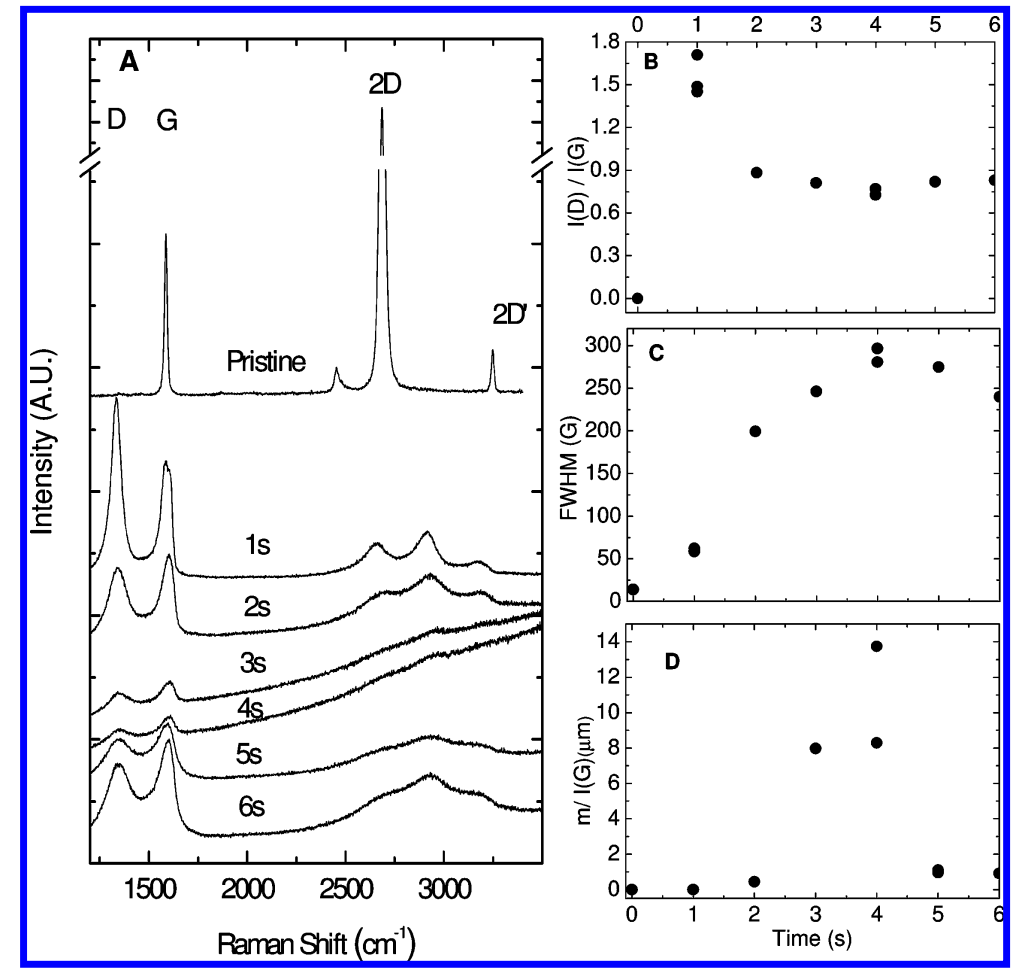

Figure 2. Raman characterization as a function of treatment time. (a) Raman spectra; (b) D to G intensity ratio; (c) FWHM(G); (d) ratio PL background slope $(m)$ to $G$ peak intensity, I(G). 


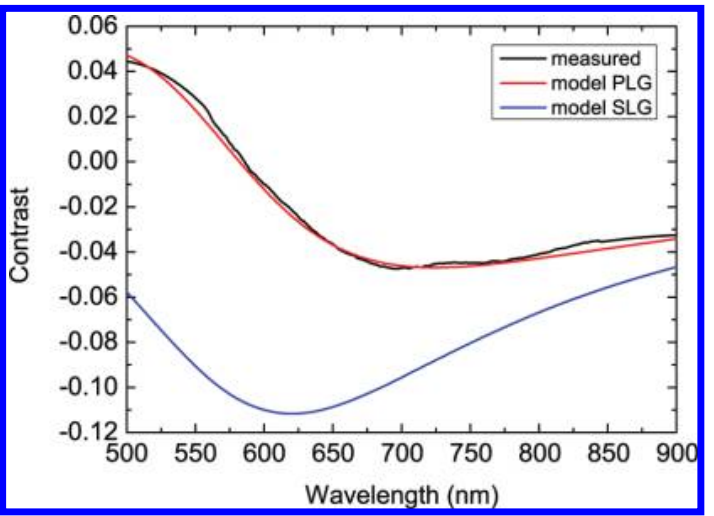

Figure 3. White-light spectrum of PLG (black line) compared to pristine graphene (blue line). ${ }^{20}$ The red line is a PLG model using a Cauchy function for the complex refractive index (see text).

tinuous $L_{\mathrm{a}}$ decrease down to $\sim 1 \mathrm{~nm}$ and a transition to a network with fewer six-fold rings for treatment longer than $1 \mathrm{~s}$. This is further validated by considering the evolution of the full width at half-maximum of the $G$ peak, FWHM(G). In defect-free graphene, a variation of $\mathrm{FWHM}(\mathrm{G})$ is observed as a consequence of doping. ${ }^{27-29}$ However, in the case of defected samples, peak broadening is a result of the activation of $\mathbf{q} \neq \mathbf{0}$ phonons. An empirical correlation between FWHM(G) and $L_{a}$ was reported in ref 30 considering a variety of disordered and amorphous carbons. Comparing $\mathrm{FWHM(G)}$ in Figure 2c with the trend in ref 30 , again we get $L_{\mathrm{a}} \sim 1 \mathrm{~nm}$ for the longest treatment. The large FWHM(G) also implies a distribution of $L_{\mathrm{a}}$ around the average value.

The ratio of the slope of the PL background $(m)$ to $I(G)$ is often used in disordered carbons as a measure of the PL strength when comparing different samples. ${ }^{31}$ We thus plot $m / I(G)$ in Figure $2 \mathrm{~d}$. This reaches a maximum, then decreases for the longest treatment, consistent with the lack of PL in ref 19 after oxygen treatment targeted at layer removing.

White-light scattering spectroscopy of PLG reveals clear differences to pristine graphene. Figure 3 illustrates the scattering spectra of both materials on $\mathrm{SiO}_{2} /$ Si. While pristine graphene appears dark throughout the spectral range covered in the experiment, corresponding to a negative interferometric contrast, ${ }^{20}$ PLG shows weaker contrast with a positive sign for wavelengths smaller than $580 \mathrm{~nm}$. The spectrum of PLG yields the complex refractive index $n^{\prime}=A_{\mathrm{n}}+B_{\mathrm{n}} / \lambda^{2}+$ $i^{*}\left(A_{\mathrm{k}}+B_{\mathrm{k}} / \lambda^{2}\right)$, with Cauchy parameters $A_{\mathrm{n}}=2.76, A_{\mathrm{k}}=$ $0.06, B_{\mathrm{n}}=3000$, and $B_{\mathrm{k}}=1500$ (assuming graphene thickness of $0.34 \mathrm{~nm}$ ), comparable to those obtained for GO. ${ }^{32}$

The data presented so far are taken for single-layer graphene (SLG). A different behavior is observed for multilayer graphene (MLG), which remains nonluminescent following treatment. Indeed, PL intensity and scattering contrast are directly correlated, as seen in Figure 4 for flakes of different thickness. The scattering contrast for treated MLG does not scale linearly with

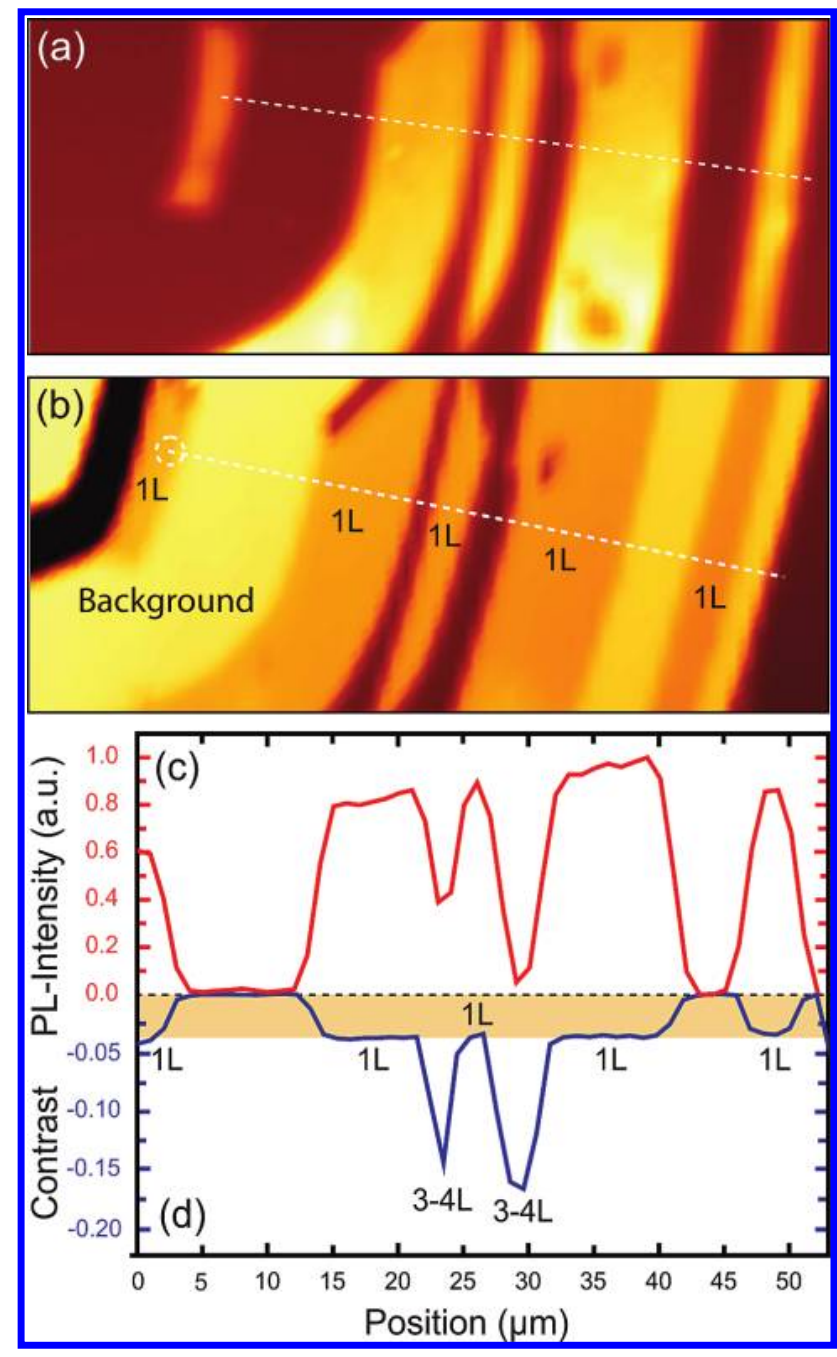

Figure 4. Correlation between PL and layer thickness. (a) PL image; (b) elastic scattering image ${ }^{20}$ of the same sample area. (c,d) Corresponding cross sections taken along the dashed lines in $(a, b) . P L$ is only observed from treated SLG, marked 1L.

the number of layers, as in the case of pristine SLG. ${ }^{20}$ The MLG spectrum only features negative contrast, while the positive contribution below $580 \mathrm{~nm}$ observed for PLG is absent. Scattering spectra from treated MLG can be represented by a superposition of treated and pristine SLG.

Oxygen plasma etching of graphite proceeds layerby-layer. ${ }^{33}$ Thus, in our case, only the topmost layer is affected. The absence of PL in MLG means that emission from the topmost layer is quenched by subjacent untreated layers. This opens the possibility of engineering sandwiched hybrid structures consisting of PLG and a variable number of pristine graphene layers.

Oxygen plasma etching is expected to yield $\mathrm{CO}$ and $\mathrm{CO}_{2}$ by successively removing carbon atoms. Etching of graphite occurs both in the basal plane and at defects. ${ }^{34}$ The latter is consistent with our observation of point-like PL features for short treatment times (Figure 1a).

It would be tempting to interpret the PL emission as coming from electron confinement in $\mathrm{sp}^{2}$ islands 


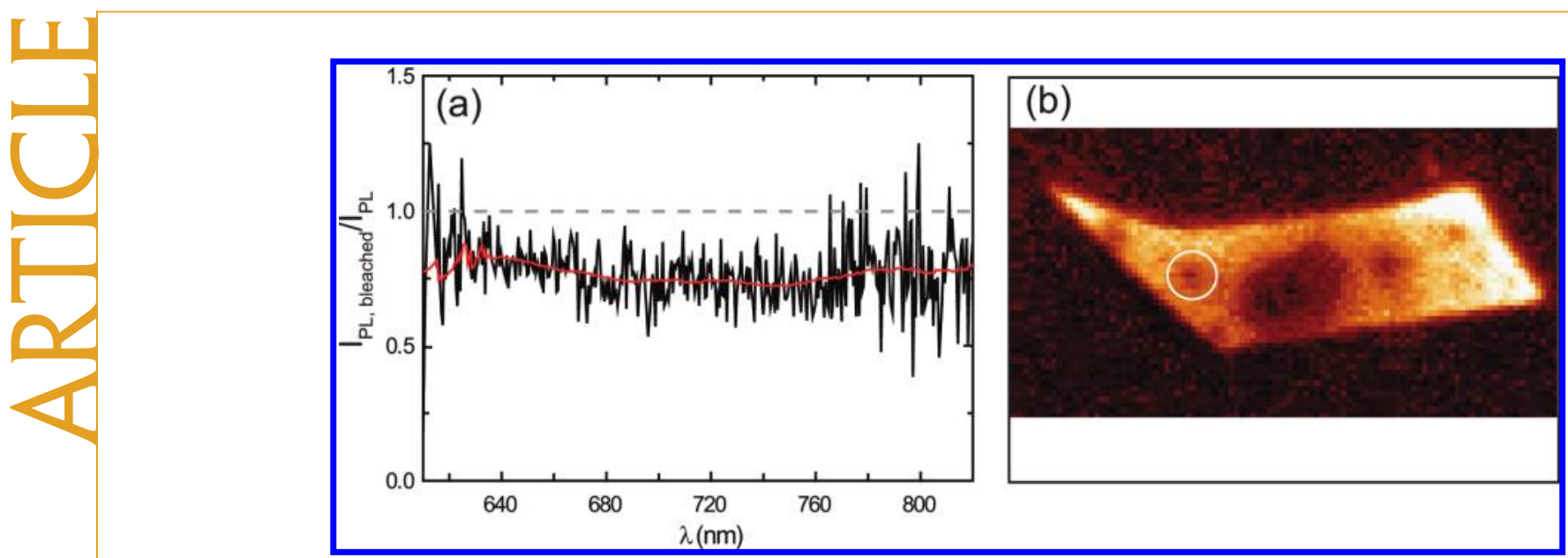

Figure 5. (a) PL intensity ratio for the area indicated by the circle in (b).

with an average size of $\sim 1 \mathrm{~nm}$, as indicated by Raman spectroscopy. Indeed, since electrons in graphene behave as massless particles, energy quantization due to confinement is expected to open a gap $\delta E \approx v_{\mathrm{F}} h / 2 d \approx$ $2 \mathrm{eVnm} / \mathrm{d}$. The resulting quantum confined energy for a quantum disk of diameter $d=2 \mathrm{~nm}$ is $1 \mathrm{eV}$. The observed emission energy distribution translates into a diameter distribution ranging from 0.94 to $1.29 \mathrm{~nm}$, in agreement with the Raman estimation. In this case, the large spectral width of the PL signal, $\sim 0.5 \mathrm{eV}$, could result from a superposition of overlapping bands with narrow linewidth centered at different size-controlled (or quantum confined) energies, corresponding to heterogeneous broadening. Then, the optical properties of the PLG would resemble those of $\pi$-conjugated polymer films, where a distribution of conjugation lengths translates into a strong inhomogeneously broadened density of states. ${ }^{35}$ At room temperature, laser irradiation in the red would lead to selective excitation of a subset of quantum confined states. Then, spectral hole burning (i.e., the selective photobleaching of this subset of homogeneously broadened lines) should be possible. This bleaching could be a photochemical modification or even a complete removal of the absorbing subset. As a result, we would observe a spectral hole, i.e., the subset absorbing a certain color would not contribute to PL. Figure 5 plots the ratio of PL measured before $\left(I_{\mathrm{PL}}\right)$ and after $\left(I_{\mathrm{PL}, \text { bleached }}\right)$ exposure to high power $(>600 \mu \mathrm{W})$ pulsed laser light at $647 \mathrm{~nm}$. The PL is measured at $530 \mathrm{~nm}$ for low excitation power $(\sim 10 \mu \mathrm{W})$. No spectral hole is observed in the detected spectral range, as would be expected for a heterogeneous ensemble of narrow bandwidth emitters. Instead, only an irreversible and uniform reduction of PL intensity occurs. For other bleaching energies in the red spectral range $(760,800 \mathrm{~nm})$, the same uniform decrease is observed, while in the blue $(473,514 \mathrm{~nm})$, the PL slightly shifts to shorter wavelength (see Figure 1c).

Thus, we find that the observed large spectral width of $0.5 \mathrm{eV}$ reflects mainly homogeneous broadening of a single emissive species, uniform across the PLG sheet. This is supported by the absence of spectral diffusion in the time-resolved data, expected for heterogeneous films ${ }^{35}$ (see Methods). If PL would indeed result from quantum confined states, ${ }^{18}$ size-related heterogeneous broadening would need to be far smaller, probably below $0.1 \mathrm{eV}$, requiring a very narrow size distribution of $\sim \pm 0.04 \mathrm{~nm}$, instead of the $\sim \pm 0.18 \mathrm{~nm}$ needed for the $0.5 \mathrm{eV}$ broadening. Since oxidation is expected to occur at different lattice sites and configurations, such high degree of ordering would seem unreasonable. Moreover, while for increasing oxidation times a successive decrease of size distribution would be expected, the spectral characteristics of the PL emission remain nearly constant. Thus, although the identification of $L_{a}$ as the quantum confinement length of massless electrons would be tempting, we rather assign the observed PL to CO-related localized electronic states at the oxidation sites.

\section{CONCLUSIONS}

We have shown that spatially uniform PL can be induced in single-layer graphene on substrates by selective plasma oxidation. Remarkably, bi- and multilayer flakes remain nonluminescent, while their elastic scattering spectra indicate the formation of sandwich-like structures containing unetched layers. The resulting photoluminescent material could pave the way toward graphene-based optoelectronics.

\section{METHODS}

Raman Background. Raman spectroscopy is a fast and nondestructive method for the characterization of carbons. These show common features in the $800-2000 \mathrm{~cm}^{-1}$ region: the $G$ and $D$ peaks. The $\mathrm{G}$ peak corresponds to the $\mathrm{E}_{2 \mathrm{~g}}$ phonon at the Bril- louin zone center. The $\mathrm{D}$ peak is due to the breathing modes of $\mathrm{sp}^{2}$ rings and requires a defect for its activation..$^{24,25,36}$ It comes from TO phonons around $\mathbf{K}^{24,25}$ is active by double resonance $(\mathrm{DR}){ }^{36}$ and is strongly dispersive with excitation energy due to a Kohn Anomaly at $\mathbf{K}^{37}$ The $2 \mathrm{D}$ peak is the second order of the 


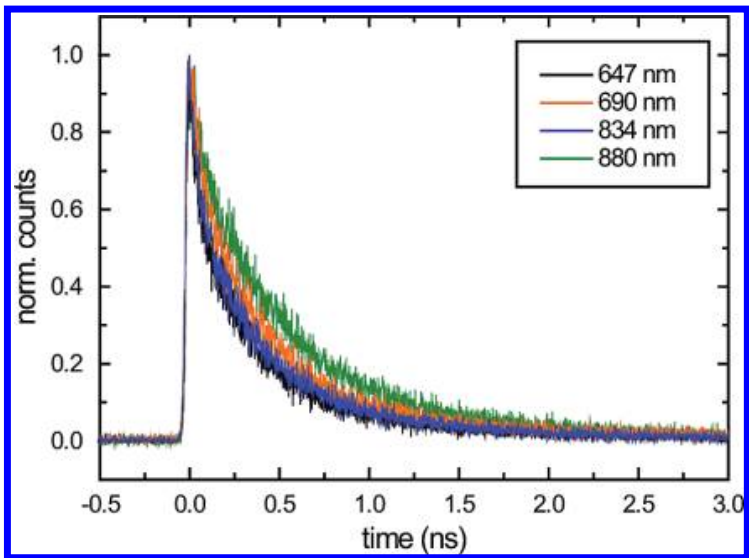

Figure 6. PL transients measured at four detection energies after excitation at $530 \mathrm{~nm}$. These are nearly uniform across the complete spectrum, indicating that spectral diffusion due to energy migration, typical for heterogeneously broadened systems, is absent.

D peak. This is a single band in monolayer graphene, whereas it splits in four in bilayer graphene, reflecting the evolution of the band structure. ${ }^{21}$ The $2 \mathrm{D}$ peak is always seen, even when no $D$ peak is present, since no defects are required for the activation of two phonons with the same momentum, one backscattering from the other. DR can also happen as intravalley process, i.e., connecting two points belonging to the same cone around $\mathbf{K}$ or $\mathbf{K}^{\prime}$. This gives rise to the $\mathrm{D}^{\prime}$ peak, $\sim 1620 \mathrm{~cm}^{-1}$ in defected graphite. The $2 D^{\prime}$ is the second order of the $D^{\prime}$ peak.

Reference 25 noted that $/(\mathrm{D}) / /(\mathrm{G})$ varies inversely with the cluster size, $L_{a}$, in poly- and nanocrystalline graphites: $/(D) / /(G)$ $=C(\lambda) / L_{a}$, where $C(514.5 \mathrm{~nm}) \sim 4.4 \mathrm{~nm} .{ }^{25,26}$ The original idea was to link $I(D)$ to phonon confinement. The intensity of the nonallowed $D$ peak would be ruled by the defect-induced lifting of the Raman fundamental selection rule. Assuming that graphite becomes uniformly nanocrystalline, the $D$ peak evolution can be estimated using Heisenberg indetermination principle: I(D) $\propto \Delta q$, with $\Delta q \Delta x \propto \hbar$ and $\Delta x \sim L_{a}$. We now know that the $\mathrm{D}$ peak activation is due to $D R$ and not to phonon confinement. However, also in this case, the higher the number of defects, the higher the chance of phonon-defect scattering and, thus, the higher I(D). Again, since the G peak is not defect-activated, even within DR, one can expect TK to hold. Now $L_{a}$ is an average interdefect distance, instead of a cluster size. TK holds until a critical defect density. Since the $D$ peak requires the presence of six-fold rings, when the network starts losing them, $/(D)$ decreases with decreasing $L_{\mathrm{a}}{ }^{24}$ In this case, $/(\mathrm{D}) / /(\mathrm{G})=C^{\prime}(\lambda) L_{\mathrm{a}}{ }^{2}$, with $C^{\prime}(514.5 \mathrm{~nm})$ $\sim 0.55 \mathrm{~nm}^{-2}{ }^{24}$ This is a very simple picture, which has proven effective to compare graphitic samples for increasing disorder. ${ }^{24,30,31}$ However, we note that a complete theory for the $D$ and $G$ Raman intensity and their dependence on the number of defects is still lacking.

PL Transients. Figure 6 plots the PL transients measured at four different detection energies after excitation at $530 \mathrm{~nm}$. All can be modeled by a triexponential decay with decay constants of 40,250 , and 1000 ps, with different relative contributions. Remarkably, there is no direct correlation between emission energy and decay dynamics, as could be expected for heterogeneously broadened systems. In this case, spectral diffusion due to energy migration would lead to faster decay in the blue spectral range and a delayed signal rise on the same time scale in the red. ${ }^{35}$ The decay traces can also be modeled using a stretched-exponential function.

Acknowledgment. A.C.F., K.S.N., and A.K.G. thank the Royal Society and the European Research Council (Grants NANOPOTS and GRAPHENE), A.H. the Deutsche Forschungsgemeinschaft (DFG-grant HA4405/4-1) and Nanosystems Initiative Munich (NIM), A. L. from the University of Palermo, Italy.

\section{REFERENCES AND NOTES}

1. Novoselov, K. S.; et al. Electric Field Effect in Atomically Thin Carbon Films. Science 2004, 306, 666-669.

2. Novoselov, K. S.; et al. Two Dimensional Gas of Massless Dirac Fermions in Graphene. Nature 2005, 438, 197-200.

3. Zhang, Y.; Tan, Y. W.; Stormer, H. L.; Kim, P. Experimental Observation of The Quantum Hall Effect and Berry's Phase in Graphene. Nature 2005, 438, 201-204.

4. Du, X.; Skachko, I.; Barker, A.; Andrei, E. Y. Approaching Ballistic Transport in Suspended Graphene. Nat. Nano 2008, 3, 491-495.

5. Han, M. Y.; Oezylmaz, B.; Zhang, Y.; Kim, P. Infrared Spectroscopy of Landau Levels of Graphene. Phys. Rev. Lett. 2007, 98, 206805-4.

6. Chen, Z.; Lin, Y. M.; Rooks, M.; Avouris, Ph. Graphene Electronics. Physica E 2007, 40, 228-232.

7. Lin, Y. M.; et al. Operation of Graphene Transistors at Gigahertz Frequencies. Nano Lett. 2009, 9, 422-426.

8. Bunch, J. S.; et al. Electromechanical Resonators from Graphene Sheets. Science 2007, 315, 490-493.

9. Hernandez, Y.; et al. High-Yield Production of Graphene by Liquid-Phase Exfoliation of Graphite. Nat. Nano 2008, 3, 563-568.

10. Eda, G.; Fanchini, G.; Chhowalla, M. Large-Area Ultrathin Films of Reduced Graphene Oxide as a Transparent and Flexible Electronic Material. Nat. Nano 2008, 3, 270-274.

11. Sun, Z.; Hasan, T.; Torrisi, F.; Popa, D.; Privitera, G.; Wang, F.; Bonaccorso, F.; Basko, D. M.; Ferrari, A. C. Graphene Mode-Locked Ultrafast Laser. ArXiv 0909.0457v1. Hasan, T. Sun, Z.; Wang, F.; Bonaccorso, F.; Tan, P. H.; Rozhin, A. G.; Ferrari, A. C. Nanotube-Polymer Composites for Ultrafast Photonics. Adv. Mater. 2009, 21, 3874-3899.

12. Li, X.; Wang, X.; Zhang, L.; Lee, S.; Dai, H. Nanoribbon Semiconductors Chemically Derived, Ultrasmooth Graphene. Science 2008, 319, 1229-1232.

13. Stampfer, C.; et al. Tunable Coulomb Blockade in Nanostructured Graphene. Appl. Phys. Lett. 2008, 92, 012102-2.

14. Ponomarenko, L. A.; et al. Chaotic Dirac Billiard in Graphene Quantum Dots. Science 2008, 320, 356-358.

15. Elias, D. C.; et al. Control of Graphene's Properties by Reversible Hydrogenation: Evidence for Graphane. Science 2009, 323, 610-613.

16. Park, S.; Ruoff, R. S. Chemical Methods for the Production of Graphenes. Nat. Nanotechnol. 2009, 4, 217-224.

17. Sun, X.; et al. Nano-Graphene Oxide for Cellular Imaging and Drug Delivery. Nano Res. 2008, 1, 203-212.

18. Luo, Z.; et al. Photoluminescence and Band Gap Modulation in Graphene Oxide. Appl. Phys. Lett. 2009, 94, 111909-3.

19. Liu, L. W.; et al. Graphene Oxidation: Thickness-Dependent Etching and Strong Chemical Doping. Nano Lett. 2008, 8 , 1965-1970.

20. Casiraghi, C.; et al. Rayleigh Imaging of Graphene and Graphene Layers. Nano. Lett. 2007, 7, 2711-2717.

21. Ferrari, A. C.; et al. Raman Spectrum of Graphene and Graphene Layers. Phys. Rev. Lett. 2006, 97, 187401-4.

22. Gokus, T.; et al. Exciton Decay Dynamics in Individual Carbon Nanotubes at Room Temperature. Appl. Phys. Lett. 2008, 92, 153116-3.

23. Lormes, W.; Hundhausen, M.; Ley, L. Time Resolved Photoluminescence of Amorphous Hydrogenated Carbon. J. Non-Cryst. Solids 1998, 227-230, 570.

24. Ferrari, A. C.; Robertson, J. Interpretation of Raman Spectra of Disordered and Amorphous Carbon. Phys. Rev. B 2000 61, 14095-14107. Ferrari, A. C.; Robertson, J. Resonant Raman Spectroscopy of Disordered, Amorphous, and Diamond-like Carbon. Phys. Rev. B 2001, 64 $075414-075426$

25. Tuinstra, F.; Koenig, J. L. Raman Spectrum of Graphite. J. Chem. Phys. 1970, 53, 1126

26. Knight, D. S.; White, W. B. Characterization of Diamond Films by Raman Spectroscopy. J. Mater. Res. 1989, 4, 385393.

VOL. 3 - NO. $12 \cdot 3963-3968 \cdot 2009$ 
27. Casiraghi, C.; et al. Raman Fingerprint of Charged Impurities in Graphene. Appl. Phys. Lett. 2007, 91, 233108-3.

28. Das, A.; et al. Monitoring Dopants by Raman Scattering in an Electrochemically Top-Gated Graphene Transistor. Nat. Nano 2008, 3, 210-215.

29. Pisana, S.; et al. Breakdown of the Adiabatic BornOppenheimer Approximation in Graphene. Nat. Mater. 2007, 6, 198-201.

30. Ferrari, A. C.; Rodil, S. E.; Robertson, J. Interpretation of Infrared and Raman Spectra of Amorphous Carbon Nitrides. Phys. Rev. B 2003, 67, 155306-155325.

31. Casiraghi, C.; Ferrari, A. C.; Robertson, J. Raman Spectroscopy of Hydrogenated Amorphous Carbons. Phys. Rev. B 2005, 72, 085401-085414.

32. Jung, l.; et al. Characterization of Thermally Reduced Graphene Oxide by Imaging Ellipsometry. J. Phys. Chem. C 2008, 112, 8499-8506.

33. You, H. X.; Brown, N. M. D.; Al-Assadi, K. Radio-Frequency (RF) Plasma Etching of Graphite with Oxygen: A Scanning Tunnelling Microscope Study. Surf. Sci. 1993, 284, 263-272.

34. Eggito, D.; Emmi, F.; Horwath, R. S.; Vukanovic, V. Plasma Etching of Organic Materials. I. Polyimide in $\mathrm{O}_{2}-\mathrm{CF}_{4}$. J. Vac. Sci. Technol, B 1985, 3, 893.

35. Meskers, S. C. J.; Hubner, J.; Oestreich, M.; Bässler, H. Dispersive Relaxation Dynamics of Photoexcitations in a Polyfluorene Film Involving Energy Transfer: Experiment and Monte Carlo Simulations. J. Phys. Chem. B 2001, 105, 9139-9149.

36. Thomsen, C.; Reich, S. Double Resonant Raman Scattering in Graphite. Phys. Rev. Lett. 2000, 85, 5214-5217.

37. Piscanec, S.; Lazzeri, M.; Mauri, F.; Ferrari, A. C.; Robertson, J. Kohn Anomalies and Electron-Phonon Interactions in Graphite. Phys. Rev. Lett. 2004, 93, 185503-4. 